\title{
Mindfulness-Based Group Approach for Undergraduate Students with Disordered Eating or Body Image Issues: A Case Study
}

Olivia Stumpf, DNP, APRN, PMHNP-BC

High and Grounded: A Wellness Practice

\begin{abstract}
Background: Mindfulness can be defined as "consciously bringing awareness to the present moment by focusing non-judgmentally on cognitions, emotions, and physical sensations" (Kabat-Zinn, 1994). This technique is applicable to patients with disordered eating, body image issues, or eating disorders (EDs) because these individuals have a tendency to become detached from their emotions and use the over or under consumption of food to regulate their internal state (Corstorphine, 2006). Mindfulness techniques are used to create a connection between thoughts, feelings and physical sensations, and external experiences. Practicing mindful eating is one way undergraduate students can be attentive to hunger cues and ensure balanced nutrition amidst a full academic schedule.

Aim: The aim of the study was to develop and evaluate a mindfulness-based group approach to managing eating disorders in college students with disordered eating or body image issues.

Methods: Undergraduate students ages 18 to 22 years old who were already receiving treatment for ED symptomatology from a university Counseling \& Psychological Services Center were recruited to participate in a six-week mindfulness-based group approach to eating awareness. Results from the progress of two participants' process observations are shared.

Results: Increases were seen in participants' confidence in using mindfulness-based practices to foster a sense of acceptance, nurturance, and adaptive behaviors.

Conclusions: A mindfulness-based group approach to treatment of disordered eating or body image issues shows promise for improving the quality of life for college-aged students. Undergraduate institutions have the advantage of using social interaction to facilitate healthy behavioral change. Future research with larger and more diverse samples is suggested, and implications regarding practice and education are also discussed.
\end{abstract}

Submitted 29 August 2017: accepted 16 October 2017

Keywords: mindfulness, group therapy, disordered eating, body image, eating disorders

Over the past few decades society has placed an increasing emphasis on physical appearance. Compounded by genetic and psychological factors, as well as environmental influences, more adolescents and young adults are developing a negative body image, or a distorted perception of body shape accompanied by feelings of dissatisfaction and critical thoughts (National Eating Disorders Collaboration, 2017). Those with body image concerns are more likely to practice disordered eating habits and develop an eating disorder in attempt to address such concerns (National Eating Disorder Association, 2016). Eating Disorders (EDs) have been cited as one of the most deadly mental health conditions 
(Academy of Eating Disorders, n.d.). The Academy of Eating Disorders (AED) reports a $0.9 \%$ lifetime prevalence of teens, young adult and adult women suffering from anorexia nervosa (AN). 1.5\% of that same population is struggling with bulimia nervosa $(\mathrm{BN})$ and 3.5\% of those individuals are dealing with binge eating disorder (BED; Academy of Eating Disorders, n.d.). Such statistics are suspected to be higher in actuality because of the many cases that go unreported (Academy of Eating Disorders, n.d.). Due to the increasing occurrence of EDs and the uniqueness of each patient and his or her condition, additional and effective treatment modalities are needed to enhance recovery. The purpose of the study was to develop and evaluate a mindfulness-based group approach to managing eating disorders in college students with disordered eating or body image issues.

\section{BACKGROUND}

Mindfulness is defined as "consciously bringing awareness to the present moment by focusing non-judgmentally on cognitions, emotions and physical sensations" (Kabat-Zinn, 1994). This technique is particularly applicable to patients with disordered eating, body image issues, or diagnosed EDs because these individuals typically have difficulty navigating such areas in a conceptual manner (Corstorphine, 2006). They become detached from their negative emotions and use the over or under consumption of food to regulate their internal state (Corstorphine, 2006). Mindfulness techniques such as body scanning, mini-meditations, mindful stretching, and the examination of metaphors are used to create a connection between the cognitive, emotional and physical experience in order to promote adaptive behavior. Instead of acting impulsively to quell distressing feelings, mindfulness encourages individuals to engage in the present moment and identify potential triggers (Kristeller, Baer, \& Quillian-Wolever, 2006). Recent years have witnessed a growing interest in the application of mindfulness-based interventions as a treatment for EDs, including AN (Heffner, Sperry, Eiferts, \& Detweiler, 2002), BN (Safer, Telch, \& Agras, 2001; Proulx, 2008), and BED (Safer, Lock, \& Couturier, 2007; Kristeller \& Hallett, 1999; Baer, Fischer, \& Huss, 2005), with results demonstrating effectiveness in eating disorder (ED) symptom reduction.

Hepworth (2011) conducted an influential study in the area of mindfulness-based eating awareness and its application as an adjunct to outpatient ED treatment. Working with six groups, each comprised of three to nine participants, Hepworth (2011) applied mindfulness principles of dialectical behavioral therapy and the work of Kausman (1998), which included the exploration of dieting, the concept of "non-hungry" eating, and eating awareness using nutrition knowledge and intuition cues. Pre- and post-group Eating Attitude Test-26 (EAT-26) scores, which evaluated dieting, food preoccupation and oral control, revealed statistically significant reductions in these domains (Hepworth, 2011). Results are indicative of the benefits of a mindfulness-based eating awareness group as an adjunct to outpatient treatment with patients presenting with varying ED symptomatology (Hepworth, 2011). However, past research has indicated that aspects of psychological well-being, such as quality of life (QOL) may be a better indicator of change than the evaluation of physiological constructs, such as those encompassed by EAT-26 (Hepworth \& Paxton, submitted). Thus, the use of an alternate measurement tool is warranted in future studies. In addition, with previous research citing the effectiveness of mindfulness in enhancing emotional regulation (Baer et al., 2005; Smith, Shelley, Leahigh, \& Vanleit, 2006), Hepworth (2011) chose a different focus, which aimed to improve the recognition of hunger and satiety cues, and use of mindfulness surrounding the relationship between food and feelings. Hepworth (2011) proposed that future studies continue to place emphasis on such topics.

Mindfulness-based eating awareness serves as a powerful tool that encourages individuals to develop a nonjudgmental awareness of their cognitions, emotions and physical sensations (Kristeller et al., 2006). EDs are 
characterized by a detachment from feelings and a focus on physical appearance and critical thoughts. Such powerful symptomatology has rippling effects on quality of life, which impacts physical well-being, psychological health and social functioning. Incorporating the use of a mindfulness-based eating awareness group into traditional eating disorder treatment programs provided on collegiate campuses can provide students with an opportunity to gain greater control over their thoughts, become more aware of their feelings and enable them to better listen to the needs of their bodies.

\section{DEFINITION OF TERMS}

Eating Disorders (EDs): EDs are characterized by abnormal eating habits that can threaten life. EDs are comprised of several different subtypes including anorexia nervosa, bulimia nervosa, and binge eating disorder. Anorexia nervosa (AN) is the failure to maintain body weight appropriate for age, sex and development due to restricted energy intake, an intense fear of gaining weight and the presence of body image disturbance. Bulimia nervosa (BN) is the occurrence of binge-eating episodes accompanied by purging and non-purging compensatory behaviors at least once a week for three months. Binge eating disorder (BED) is described as recurrent binge eating without compensatory behavior accompanied by a sense of loss of control (Grohol, 2013).

Disordered eating: Disordered eating is defined as skipping meals, dieting, self-induced vomiting, over-exercising, diuretic or laxative use, and compulsive eating. It includes irregular and chaotic eating patterns (Eating Disorders Victoria, 2016).

Body image: Body image refers to perceptions, beliefs and feelings regarding the subjective picture of one's own body (National Eating Disorder Information Centre, 2014).

Mindfulness-based eating awareness: Mindfulness-based eating awareness blends mindful eating exercises, such as being aware of hunger, chewing food slowly, tuning into taste and noticing fullness, with mindful meditation practice to cultivate moment-to-moment awareness of self (Novotney, 2012).

Standard of care: Standard of care received by patients who suffer from EDs on an inpatient or outpatient basis includes psychoeducation regarding health and disease processes, as well as nutrition counseling. Group therapy focuses on body acceptance, self-esteem, exploration of food and feelings, promotion of mind over mood, and enhancement of coping and assertiveness skills. Alternative therapies include equine, art, music, and dance therapies. Central to treatment is psychotherapy ranging from family therapy, cognitive-behavioral therapy and dialectical behavioral therapy (Academy of Eating Disorders, n.d.).

\section{METHODS}

With IRB approval, this case study targeted undergraduate females ages 18 to 22 years, who were referred to the eating disorder treatment team at the Counseling and Psychological Services Center at a university. Participants were identified by the team as struggling with disordered eating or body image issues and would be followed by the team during the duration of the group. Individuals with prior exposure to mindfulness practices or those with a diagnosed 
DSM Axis-II disorder, or a lifelong personality or intellectual disorder, were unable to participate due to influential knowledge or compounding comorbidity factors.

The eating disorder treatment team was comprised of mental health professionals at the Counseling and Psychological Services Center and medical professionals at the Student Health Center. Students referred to the eating disorder treatment team were screened and evaluated in order to determine the appropriate care needed. The team identified students with disordered eating or body image issues as previously defined in order to recruit participants. In addition to the standard of care deemed appropriate by the treatment team, those identified as potential participants, were followed by the team during the duration of the group.

Demographic information was collected on age, race, current diagnoses, hospitalization history, family background, and past experience with mindfulness practices. The participants partook in one-hour mindfulness-based group work sessions once weekly for six consecutive weeks. The author and primary investigator, a Nursing Supervisor at a residential center for adolescents with EDs, and educated in mindfulness-based eating awareness, coled the group sessions in conjunction with a mental health professional from the Counseling \& Psychological Services Center and member of the eating disorder treatment team. Throughout the experience, the primary investigator maintained notes in the form of process observations in order to further evaluate overall effects of group work.

\section{MINDFULNESS-BASED GROUP}

Each week for six weeks, tenants of mindfulness were introduced to facilitate awareness of cognitions, emotions and physical sensations, including hunger and fullness cues. Relevant readings from Johnston's (2000), Eating in the Light of the Moon, were also incorporated into discussion to further explore mindfulness principles. Examples include myths, metaphors and storytelling on topics such as the woman spirit, hunger as a metaphor, nourishment and intuition that encouraged participants to seek present balance in their lives (Johnston, 2000). Group sessions began and concluded with mindful breathing, a mindful mini-meditation or a forgiveness mediation. Mindful breathing is intended to relax the body in order to promote access to the awareness of physiological processes (Kristeller, 2003). Mindful mediations are more focused, as they ask participants to be cognizant of their bodies in the everyday environment (Kristeller, 2003). The use of mindful meditations and forgiveness meditations, or the exploration and acceptance of feelings in a nonjudgmental fashion, seek to unite the cognitive, emotional and physical resulting in awareness and cultivation of inner wisdom (Kristeller, 2003). See Figure 1. 
Figure 1: Group Work Outline

\begin{tabular}{|c|c|c|}
\hline Session Number: & Group Work Phase: & Session Description: \\
\hline Session One & Warm -Up & $\begin{array}{l}\text {-Group set-up and objectives } \\
\text {-Consensus agreement } \\
\text {-Pre-Group EDQLS } \\
\text {-First mindfulness component: } \\
\text { Mental, emotional and physical } \\
\text { presence } \\
\text {-Mindful breathing }\end{array}$ \\
\hline Session Two & Action & $\begin{array}{l}\text {-Second mindfulness component: } \\
\text { Internal versus external wisdom } \\
\text {-Exploring the woman spirit } \\
\text {-Progressive muscle relaxation }\end{array}$ \\
\hline Session Three & Action & $\begin{array}{l}\text {-Third mindfulness component: } \\
\text { Non-judgmental awareness } \\
\text {-Hunger and fullness awareness } \\
\text {-Mindful meditation }\end{array}$ \\
\hline Session Four & Action & $\begin{array}{l}\text {-Fourth mindfulness component: } \\
\text { Free habitual thinking, feeling } \\
\text { and action } \\
\text {-Symbolism (hunger as a } \\
\text { metaphor) } \\
\text {-Nourishment (physical versus } \\
\text { emotional) } \\
\text {-Mindful eating meditation }\end{array}$ \\
\hline Session Five & Action & $\begin{array}{l}\text {-Fifth mindfulness component: } \\
\text { Balance choice, wisdom and } \\
\text { acceptance } \\
\text {-Intuition for decision making } \\
\text {-Developing bodily wisdom } \\
\text {-Acknowledging feelings } \\
\text {-Mindful meditation }\end{array}$ \\
\hline Session Six & Concluding & $\begin{array}{l}\text {-Summary } \\
\text {-Celebration and reflection } \\
\text {-Feedback and post-group } \\
\text { EDQLS } \\
\text {-Forgiveness meditation }\end{array}$ \\
\hline
\end{tabular}




\section{RESULTS}

Four female undergraduate students ranging from 18 to 21 years old with a mean age of 19.25 years were identified and simultaneously followed by the eating disorder treatment team for disordered eating or body image issues. Of the 4 participants, 2 failed to attend all six sessions. Thus, both participants were excluded from the study. The remaining 2 participants reported their race as White.

Throughout the course of the warm-up, action and concluding stages of the group, recorded process observations yielded important findings. Overall, members demonstrated little resistance and denial, as they expressed interest in self-exploration and investment in ED symptom reduction through participation in group work. Although members rarely challenged leaders or other group members, identification with one another regarding shared struggles surrounding ED symptomatology enhanced interpersonal relationships and facilitated interpersonal learning within the group. In particular, group members expressed feeling comfortable with self-disclosure, which prompted mutual support, problem solving and instillation of hope as they were presented with daily challenges and food-focused holidays, such as Thanksgiving and Christmas. Furthermore, during group sessions, co-leaders played moderately active roles. They did so by linking statements to facilitate group cohesion, highlighting insightful aspects of group process to promote effective work, and encouraging self-exploration among members to foster understanding of the relationship between emotions, cognitions and physical sensations.

\section{DISCUSSION}

Process observations recorded throughout the course of the group yielded valuable information from participants. Members expressed gratitude in having the opportunity to reveal vulnerabilities in a safe environment with others who share similar struggles. They also expressed appreciation for the imparting of information regarding mindfulnessbased eating awareness. They conveyed accomplishment and confidence in using these practices to foster acceptance, nurturance, and adaptive behavior. Such interpersonal learning is the foundation of group work, which supports positive group process and outcome, and adds to its therapeutic value (Wanlass, Moreno \& Thomson, 2005). Additional sessions may have provided the opportunity for advanced interpersonal learning and growth by allowing group members to embody different group roles, experience confrontation, express deeper rooted feelings, practice communication skills, and test new behaviors. Identification of additional unfamiliar feelings and exploration of their relation to cognitions and physical sensations could have more greatly enhanced insight and further impacted overall QOL. Despite such confines, mindfulness-based group work as an additive to standard care shows potential as a supportive tool for undergraduates experiencing disordered eating or body image issues. The time-limited nature of the group placed restrictions on treatment outcomes by preventing students from potentially gaining further benefit with additional sessions.

\section{LIMITATIONS}

Several limitations were encountered through this case study examining the effects of a mindfulness-based group approach on undergraduates with disordered eating or body image issues. The small sample size due to low participant attendance prevented the establishment of a sample that was representative of the full range of disordered eating or body image issues and associated diagnoses present in the targeted population. 
The sample was gathered based on referral. Participants were to be under the care of the eating disorder treatment team at the Student Counseling \& Psychological Services Center and deemed appropriate for group work based on disordered eating or body image symptomatology. Furthermore, they were to continue with the care advised by the treatment team during the duration of the study. In addition, in order to ensure consistency of intervention replication, the mindfulness-based group work approach followed established tenants of mindfulness-based eating awareness. As such, a weekly outline of specific topics and exercises to be covered was standardized.

\section{CONCLUSIONS}

Additional research is needed to determine the implications for use of a mindfulness-based group work approach in college settings. In particular, future investigation should aim for a larger and more ethnically diverse sample size in order to detect the existence of statistically significant improvements in participants as a result of mindfulness-based group work. Additional research regarding relationships between QOL scores and variables such as primary diagnosis, weight, motivation for change and ED symptom severity in an expanded undergraduate population is warranted. Thus, although the use of a mindfulness-based group work approach shows promise in facilitating the recovery process, additional evidence is needed.

With approximately $14 \%$ of undergraduate females and $4 \%$ of undergraduate males receiving a positive screen for a current eating disorder, necessity for further research is supported by the need for additional services (Eisenberg, Nicklett, Roeder, \& Kirz, 2011). With attainment of independence, individuality, academic success and peer inclusions in the forefront of many undergraduate concerns, physical, social and emotional well-being play an important role in achievements and overall functioning. However, when experiencing navigational difficulty in areas of intra- and interpersonal conflict, underlying disordered eating or body image issues may present. As a result, trouble with emotional regulation and problem solving skills can make daily functioning a challenge. Therefore, college campuses become pivotal, but ample environments to combat disorder development and progression by fostering positive change (Eisenberg et al., 2011). As a community that provides opportunities for intellectual learning and emotional growth, undergraduate institutions have the advantage of using social interaction to facilitate healthy behavioral change that has the ability to radiate into all aspects of life.

With the ED field continuing to refine existing and expanding upon new psychological treatments, mindfulness has the potential to be a useful component in the management of the cognitive distortion and resulting maladaptive behavior that individual and group cognitive behavior therapy seek to unravel. With the aims of promoting emotional awareness and balance, with the reduction of thoughts surrounding disordered eating and related behaviors, mindfulness as an aspect of group cognitive behavior therapy for undergraduates holds promise. However, in order to prevent barriers to providing such care, adequate communication and collaboration amongst treatment team members can ensure the creation of an environment where recovery can be fostered (Schaffner \& Buchanan, 2008). In addition, proper mindfulness training can equip group leaders with the necessary expertise to respond appropriately to the unique dynamics of the milieu. Given its established low cost for implementation, group therapy for EDs will continue to be incorporated into evidence-based treatment plans (Kass, Kolko, \& Wifley, 2013). Enduring efforts to effectively train, disseminate and implement ED group therapy interventions will 
facilitate increased access to evidence-based care and ensure its sustainability. Use of group work approaches, including mindfulness-based interventions will help guide the development of a present and nonjudgmental understanding of the self while cultivating deep human connections with others in order to create long-lasting change. 


\section{REFERENCES}

Academy of Eating Disorders. (n.d.). Resources for Eating Disorders. Retrieved March 1, 2014, from Academy of Eating Disorders website: https://www.aedweb.org/index.php/education/eating-disorder-information/ eating-disorder-information-2

Baer, R. A., Fischer, S., \& Huss, D. B. (2005). Mindfulness-based cognitive therapy applied to binge eating: A case study. Cognitive and Behavioral Practice, 12(3), 351-358. https://doi.org/10.1016/S1077-7229(05)80057-4

Corstorphine, E. (2006). Cognitive-emotional-behavioural therapy for Eating Disorders: working with beliefs about emotions. European Eating Disorders Review, 14, 448-461. https://doi.org/10.1002/erv.747

Eating Disorders Victoria (2016). Disordered eating and dieting. Retrieved November 1, 2017, from Eating Disorders Victoria website: https://www.eatingdisorders.org.au/eating-disorders/disordered-eating-a-dieting

Eisenberg, D., Nicklett, E. J., Roeder, K., \& Kirz, N. E. (2011). Eating disorder symptoms among college students: Prevalence, persistence, correlates, and treatment-seeking. Journal of American College Health, 59(8), 700-707. https://doi.org/10.1080/07448481.2010.546461

Heffner, M., Sperry, J., Eiferts, G.H., \& Detweiler, M. (2002). Acceptance and commitment therapy in the treatment of an adolescent female with anorexia nervosa: A case example. Cognitive and Behavioral Practice, 9, 232-236. https://doi.org/10.1016/S1077-7229(02)80053-0

Hepworth, N.S. (2011). A Mindful Eating Group as an Adjunct to Individual Treatment for Eating Disorders: A Pilot Study. Eating disorders, 19(1), 6-16. https://doi.org/10.1080/10640266.2011.533601

Hepworth, N.S., \& Paxton, S.J. (Submitted). Treatment perceptions in bulimia nervosa and binge eating disorder: A concept mapping approach.

Grohol, J.M. (2013). DMS-5 Changes: Feeding and Eating Disorders. Retrieved November 1, 2017, from PsychCentral website: https://pro.psychcentral.com/dsm-5-changes-feeding-eating-disorders/004412.html

Johnston, A. (2000). Eating in the Light of the Moon. Carlsbad, CA: Gurze Books.

Kabat-Zinn, J. (1994). Wherever you go, there you are: Mindfulness meditation in everyday life. New York, NY: Hyperion.

Kass, A.E., Kolko, R.P., \& Wifley, D.E. (2013). Psychological treatments for eating disorders. Curr Opin Psychiatry, 26(6), 549-555. https://doi.org/10.1097/YCO.0b013e328365a30e

Kausman, R. (1998). If not dieting, then what? New South Wales, Australia: Allen \& Unwin.

Kristeller, J.L. (2003). Mindfulness, wisdom, and eating: Applying a multi-domain model of meditation effects. Constructivism in the Human Sciences, 8(2), 107-118. Retrieved from http://www.metanexus.net/archive/conference2004/pdf/kristeller.pdf

Kristeller, J.L., Baer, R.A., \& Quillian-Wolever, R. (2006). Mindfulness-based approaches to eating disorders. In R. Baer (Ed.), Mindfulness and acceptance based interventions: Conceptualization, application and empirical support (pg. 75-89). San Diego, CA: Elsevier. https://doi.org/10.1016/B978-012088519-0/50005-8 
Kristeller, J. L., \& Hallett, C. B. (1999). An exploratory study of a meditation-based intervention for binge eating disorder. Journal of Health Psychology, 4, 357-363. https://doi.org/10.1177/135910539900400305

National Eating Disorder Association. (2016). What Is Body Image? Retrieved October 9, 2017, from NEDA website: https://www.nationaleatingdisorders.org/what-body-image

National Eating Disorder Information Centre. (2014). Body Image \& Self Esteem. Retrieved February 16, 2015, from National Eating Disorder Information Centre website: http://www.nedic.ca/know-facts/body-image-selfesteem

National Eating Disorders Collaboration. (2017). What is Body Image? Retrieved November 1, 2017, from National Eating Disorders Collaboration website: http://nedc.com.au/body-image

Novotney, A. (2012). Bite, chew, savor. Monitor on Psychology, 43(10), 42.

Proulx, K. (2008). Experiences of women with bulimia nervosa in a mindfulness based eating disorder treatment group. Eating Disorders, 16, 52-72. https://doi.org/10.1080/10640260701773496

Safer, D.L., Lock, J., \& Couturier, J.L., (2007). Dialectical behavior therapy modified for adolescent binge eating disorder: A case report. Cognitive and Behavioral Practice, 14, 157-167.

https://doi.org/10.1016/j.cbpra.2006.06.001

Safer, D.L., Telch, C.F., \& Agras, W.S. (2001). Dialectical behavior therapy adapted for bulimia: A case report. International Journal of Eating Disorders, 30, 101-106. https://doi.org/10.1002/eat.1059

Schaffner, A. I., \& Buchanan, L. P. (2008). Integrating evidence-based treatments with individual needs in an outpatient facility for eating disorders. Eating Disorders: The Journal of Treatment \& Prevention, 16(5), 378-392. https://doi.org/10.1080/10640260802370549

Smith, B. W., Shelley, B. M., Leahigh, L., \& Vanleit, B. (2006). A preliminary study of the effects of a modified mindfulness intervention on binge eating. Complementary Health Practice Review, 11(3), 133-143. https://doi.org/10.1177/1533210106297217

Wanlass, J., Moreno, J. K., \& Thomson, H. M. (2005). Group therapy for eating disorders: A retrospective case study. Journal for Specialists in Group Work, 30(1), 47-66. https:/ /doi.org/10.1080/01933920590908697

Address author correspondence to:

Olivia Stumpf, DNP, APRN, PMHNP-BC

Co-Founder, Psychiatric Nurse Practitioner

High and Grounded: A Wellness Practice

4816 N. Woodmere Fairway \#1012, Scottsdale, AZ 85251

dr.olivia@highandgroundedwellness.com 